\title{
escrito pormu
}

\section{${ }^{1}$ Claudia Sandra Krmpotic}

Investigadora del Consejo Nacional de Investigaciones Científicas y

Técnicas, Ciudad Autónoma de Buenos Aires, Argentina. Profesora en las Universidades Nacionales de Buenos Aires y de la Matanza en Argentina.

\claudia.k@conicet.gov.ar

(i) ORCID ID http://orcid.org/0000-0001-8969-9345

Cómo citar / citation:

Krmpotic, C. (2019). La espiritualidad en la educación del Trabajo Social en Argentina, desde la perspectiva de estudiantes avanzados. Voces desde el Trabajo Social, 7(1), 36-59. https://doi.org/10.31919/voces.v7i1.74

Recibido / received: 11 de abril de 2019
Revisado / reviewed:

9 de septiembre de 201910 de octubre de 2019

Derechos de autoría / Copyright: (C) 2019 Krmpotic, C. Este es un artículo de acceso abierto y distribuido bajo los términos de la licencia y políticas de Creative Commons Attribution 4.0 International License. 


\section{LA ESPIRITUALIDAD EN LA EDUCACIÓN DEL TRABAJO SOCIAL EN ARGENTINA, DESDE LA PERSPECTIVA DE ESTUDIANTES AVANZADOS}

Claudia Sandra Krmpotic ${ }^{1}$ iD

\section{Resumen}

$$
\text { GopenaCCEsS PeER-REVEweo }
$$

El artículo explora el interés por la espiritualidad en la formación en Trabajo Social en la Argentina, en el contexto de las tendencias halladas en el Trabajo Social internacional. A partir de los datos obtenidos a través de un cuestionario autoadministrado aplicado a 137 estudiantes avanzados de la Universidad Nacional de La Matanza (Buenos Aires, Argentina) en los cursos 2016-2017, se analizan sus opiniones y creencias en torno de tres dimensiones: a) movilización espiritual; b) la espiritualidad como dimensión del diagnóstico y de la intervención social; y c) su presencia en los contenidos curriculares como en la bibliografía de apoyo. El análisis cuantitativo de los datos interpretados a la luz de los conceptos relevados, sugieren que, si bien los participantes reconocen que la espiritualidad forma parte del universo del Trabajo Social, se integra débilmente al proceso de formación profesional, revelando históricas disyuntivas profesionales.

\section{Descriptores}

Espiritualidad, Trabajo Social, educación, calidad de vida, Argentina. 


\section{Sumario}

Introducción. Aproximaciones conceptuales. La espiritualidad en el Trabajo Social Iberoamericano. Metodología de la investigación. Visiones de los estudiantes avanzados. Discusión. Conclusiones. Referencias.

\section{[EN] SPIRITUALITY IN SOCIAL WORK EDUCATION IN ARGENTINA, FROM THE PERSPECTIVE OF ADVANCED STUDENTS}

\section{Abstract}

The article explores the interest in spirituality in Social Work training in Argentina, in the context of the trends found in international Social Work. From the data obtained through a self-administered questionnaire applied to 137 advanced students of the National University of La Matanza (Buenos Aires, Argentina) in the 2016-2017 courses, their opinions and beliefs are analyzed around three dimensions: a) spiritual mobilization; b) spirituality as a dimension of diagnosis and social intervention; and c) curricular content and supporting bibliography. Quantitative analysis of the data interpreted in the light of the surveyed concepts suggests that although participants recognize that spirituality is part of the universe of social work, weakly integrated into the training process, revealing historical professional dilemmas.

\section{Keywords}

Spirituality, Social Work; education, quality of life, Argentina. 


\section{Introducción}

Un resurgimiento del interés en la espiritualidad se constata como tendencia global en las humanidades y las ciencias sociales desde finales del siglo XX. Una prestación sociosanitaria culturalmente competente y sensible a laespiritualidad, sereconoceensintonía con un concepto renovado de calidad de vida que integra las dimensiones bio, psico, socio y espiritual. Su papel ha sido reconocido por las ciencias sociales en general (Krmpotic, 2016), y por las profesiones de cuidado en particular, como la enfermería (Morillo, González-Serna \& Llanos, 2017), la psiquiatría (Bianchi, 2013) y el Trabajo Social (Bhagwan, 2013; Crisp, 2008; Sheridan \& Amato-von, 1999; Canda, 2009, entre otros). Esta tendencia hacia visiones holísticas e integrales también se registra en el orden de las políticas de salud y de bienestar (Toniol, 2015). Responde también a la realidad de profesionales y usuarios que experimentan una pluralización de prácticas, saberes y agentes (Krmpotic \& Ponce de León, 2017).

El interrogante acerca de su incorporación al Trabajo Social local surge del trabajo de campo en el que venimos notando una tematización del pluralismo cultural y médico, como diversas combinaciones entre la actividad profesional y las creencias espirituales y terapéuticas como en el caso de una experimentada trabajadora social (Krmpotic, 2018). También respecto de vínculos entre práctica profesional y actividad pastoral, como en los casos de trabajadores sociales que desarrollan una labor técnica en comunidades adventistas y neo-pentecostales (Barrón, 2017), ampliando el campo de acción respecto del espacio tradicional y predominantemente voluntario en las organizaciones de caridad católicas. En estas situaciones, se revela la importancia del desarrollo personal (expansión del plano espiritual, cambios en los modos de vivir, etc.) como condición necesaria para una mejor interacción y productividad social, aunque ello no trasunta cabalmente en el ejercicio profesional, como tampoco en la sistematización y divulgación de dichas experiencias. Del mismo modo, es llamativa la ausencia de la temática en la formación como en las prácticas en el Trabajo Social profesional local, lo que se manifiesta en la casi nula investigación sobre el asunto.

En este contexto, el artículo presenta los alcances de una indagación exploratoria de las opiniones y creencias de estudiantes avanzados en Trabajo Social frente a este constructo, a partir de los datos obtenidos a través de un cuestionario estructurado, autoadministrado y anónimo aplicado a 137 estudiantes avanzados en Trabajo Social, de la Universidad Nacional de La Matanza (Buenos Aires, Argentina). Los 
resultados fueron interpretados a la luz de las publicaciones relevadas, y ordenados entorno detres dimensiones de análisis: a) movilización espiritual; b) la espiritualidad como dimensión del diagnóstico y de la intervención social; y c) su reconocimiento en los contenidos curriculares como en la bibliografía de apoyo.

\section{Aproximaciones conceptuales}

El inicio del nuevo siglo implicó para las ciencias sociales un llamado de atención en la relación entre espiritualidad y calidad de vida, entre el arte de convivir en sintonía con el arte de vivir. En un sentido amplio, la espiritualidad se entiende como el aspecto inmaterial, intelectual o moral del hombre. Está asociada a la creencia en la existencia de un poder sobrenatural, creador y regulador del universo, que dota al hombre de una naturaleza espiritual que continúa existiendo después de la muerte. Como señala Myers (2008), lo espiritual refiere a una capacidad humana que permite a los individuos trascender su vivencia personal, experimentar lo sagrado del universo y buscar el significado de la vida. Foucault (2008) concibe la espiritualidad como una búsqueda, una experiencia a través de la cual el sujeto se transforma. Furman, Benson, Canda \& Grimwood (2005) la consideran como una actitud hacia la vida, en búsqueda de significados y propósitos en las relaciones con uno mismo, con los demás y con la realidad del universo, la naturaleza o una presencia superior. Cervantes (2011, p. 9) nos remite a la

experiencia de sentir que eres parte de algo más grande y más profundo que tú, algo que te conecta con todo y con todos, que te hace ver a todos los seres humanos como hermanos y al planeta como lo común. Casa que tenemos que cuidar.

Para algunos refleja la búsqueda de integración de la conciencia individual y planetaria, con referencia a una conciencia política individual pero vinculada a la responsabilidad y sensibilidad ética (Torrent, 2011) o como dice De la Iglesia (2011), una espiritualidad socialmente comprometida. Si bien la espiritualidad no es sinónimo de religión, la incluye. En tanto expresión particular de la espiritualidad, refiere a prácticas y creencias institucionalizadas, a un sistema formal de fe y adoración, $y$ al reconocimiento de escrituras sagradas. En cualquier caso, y a propósito de la secularización como rasgo dominante de la modernización, seguimos a Berger (1999, p. 9) cuando afirma que "el mundo de hoy es masivamente religioso, es todo menos el mundo secularizado que había sido pronosticado (con alegría o con desánimo) por tantos analistas 
de la modernidad" (trad. propia). En algunas sociedades, el resurgimiento de la espiritualidad está asociado al reconocimiento de las formas de conocimiento tradicionales e indígenas (Bhagwan, 2013). En América Latina, la cosmovisión ancestral de la vida también tiene impacto en las creencias locales. La noción de una vida en plenitud reúne lo natural y lo espiritual y se expresa en la armonía, en el equilibrio interno y externo de una comunidad.

En Trabajo Social, autores como Dominelli (2004) se refieren indirectamente a una movilización espiritual a través de prácticas culturalmente sensibles que reconozcan la fluidez de la cultura en los estilos de vida paralelos. Sperry y Shafranske (2005) apelan a la noción de prácticas conscientes, Canda (2009) a la de práctica espiritualmente sensible, denotando cómo las visiones del mundo y las tradiciones espirituales nos afectan. Crisp (2008) considera la espiritualidad como una experiencia vivida que incluye rituales de vida, creatividad, acción social y sentido del lugar, independientemente de nuestros antecedentes religiosos. Otros autores resaltan la relación de la espiritualidad y el lenguaje, a través del cual las personas pueden identificarse y pensar críticamente (Kearney, 1990; Perrin, 2007). Como camino de autoconocimiento Jacobs (1997, p. 173) entiende que los trabajadores sociales deben tener en cuenta la dimensión espiritual en sus vidas, pues "a medida que nos involucramos en nuestro trabajo con los clientes, debemos estar conscientes de nuestras propias experiencias espirituales y religiosas. Nuestro trabajo integral con el cliente requiere que nos comprometamos a reflexionar sobre nuestro propio viaje espiritual y psicológico". Por la misma razón, algunos señalan con preocupación que en la formación profesional habitualmente se enseña a los trabajadores sociales a separar la vida espiritual de la práctica profesional (Crisp, 2008; Gilligan \& Furness, 2006; Lindsay, 2002), lo que puede en parte explicar el escaso debate sobre la cuestión en el escenario profesional.

La tendencia también se refleja en las organizaciones internacionales. La vida interior recupera preminencia en el bienestar individual y social. Así lo entendió la Organización Mundial de la Salud (OMS) cuando propuso en 1998 la incorporación del plano espiritual al concepto de calidad de vida, entendiendo que la espiritualidad, la religiosidad y las creencias personales afectan la salud y el bienestar. Ello trajo aparejado innovaciones en los cuidados y manejo del sufrimiento, en la economía de la salud como en la organización de los servicios sociales.

Asimismo, impacta en la definición global de Trabajo Social aprobada 
por la Federación Internacional de Trabajadores Sociales (IFSW) y la Asociación Internacional de Escuelas de Trabajo Social (IASSW) en julio de 2014, en la que se afirma que el desarrollo social está basado en evaluaciones e intervenciones holísticas, bio-psico-sociales y espirituales que trascienden las distinciones micro-macro. Se anticipa que las nuevas generaciones de profesionales deben estar preparados para los desafíos que ofrecen las sociedades multiculturales, lo que implica competencias para trabajar con población con diversos rasgos, culturas y creencias, de una manera bien informada y culturalmente sensible. El Trabajador Social debe abandonar los supuestos de la cultura mayoritaria para comprender a los usuarios. La perspectiva se proyecta a la educación profesional intercultural, en la necesidad de descubrir el significado de la dignidad humana y el bienestar en las conciencias, lo que resulta esencial para definir el ideal de justicia y autorrealización, entre otros aspectos (Krmpotic \& Ponce de León, 2015).

Respecto de la educación profesional y la espiritualidad, algunas investigaciones publicadas ofrecen interesantes abordajes. Entre ellas, se cuentan los trabajos de Janigová y Bednarova (2015) aplicando el cuestionario de calidad de vida orientado a la espiritualidad
(WHOQOL Spirituality, Religiousness and Personal Beliefs (SRPB) FieldTest Instrument, 2012) a una muestra intencional de 401 estudiantes del programa de estudios de Trabajo Social en Eslovaquia, Polonia, Letonia; el de Bhagwan (2013) aplicando un cuestionario a 66 profesores de Trabajo Social, en 16 universidades de Sudáfrica, y el de Sheridan \& AmatoVon (1999) empleando la escala Role of Religion and Spirituality in Practice a una muestra de 208 estudiantes de dos escuelas de Trabajo Social norteamericanas, cuyos resultados consolidan la tendencia y coinciden en la ausencia de contenidos específicos en las curriculas.

\section{La espiritualidad en el Trabajo Social Iberoamericano}

Los autores de habla hispana admiten que, aunque en la actualidad se hace hincapié en la necesidad de adoptar una visión integral de las personas con quienes intervenimos, lo espiritual se minimiza con respecto a otras dimensiones como la sociológica, la biológica, la psicológica y de la reproducción material de la vida humana. Varias razonesconvergen para explicar su invisibilidad en el Trabajo Social, en especial las relacionadas con: a) el arquetipo del ser racional que tiende a anular y controlar los aspectos emocionales y de creencias; b) las dificultades de comprensión 
del fenómeno de la espiritualidad y la religiosidad; c) la distancia que asumieron las profesiones modernas respecto de la religión como un ejercicio íntimo, privado, lo que ha reforzado sus connotaciones restrictivas. En tal sentido "existen imaginarios arraigados que menosprecian el valor de la espiritualidad, la reducen al ámbito de la religiosidad popular y la inscriben en las esferas de la metafísica, o la refieren a eventos desarticulados de la vida cotidiana" (Cáceres, Hoyos, Navarro \& Sierra, 2008, p. 381).

Sin embargo, hoy en día es imposible ignorar las estrategias espirituales desarrolladas y utilizadas por las personas a las que servimos en las difíciles circunstancias que atraviesan. Ortiz (2007) nos invita a reflexionar sobre el vínculo entre espiritualidad y Trabajo Social, a partir de conceptos como espiritualidad, religión, ética y moral, profundizando en lo que ella llama los dilemas espirituales que afectan el trabajo social: espiritualidad vs. racionalidad, espiritualidad como factor protector o como factor de riesgo, espiritualidad/ religión o manipulación política, espiritualidad androcéntrica vs. espiritualidad inclusiva, religión como alternativa de recuperación o como sectas destructivas.

Por su parte, Moñivas, García y García De Silva (2012) enfocan en una técnica como la atención plena (mindfulness) para identificar su desarrollo en el campo de las neurociencias y las psicoterapias y su proyección al Trabajo Social en diversas áreas de intervención, así como sus posibilidades en el escenario español. En este sentido, señalan que los trabajadores sociales, especialmente los de Estados Unidos y Canadá, están desarrollando una variedad de intervenciones basadas en la atención plena en diferentes contextos y poblaciones. Los autores respaldan la posición de Hick (2008) quien considera positiva su incorporación a la práctica profesional en tres niveles: el autocuidado, las intervenciones directas con individuos, familias o grupos (micro prácticas); y las intervenciones indirectas en el trabajo comunitario y en la política (mezzo y macro prácticas).

Del mismo modo, Torregrosa (2016, p. 2) considera que incluir la dimensión espiritual en el Trabajo Social es necesario para tener una visión más completa del ser humano. Considera valiosa la incorporación de

un método complementario y aplicable a la intervención profesional, como la logoterapia iniciado por Frankl (1905-1997) y una visión de la relación de ayuda como herramienta fundamental para promover el desarrollo personal de ambos, del profesional como de las personas con las que intervenimos. 
Por su parte, Fernández (2015, p. 292) ha observado que "la tendencia parece sugerir que estudiar el tema ya no es un tema tabú sino un requisito" (con referencia a los Estados Unidos y el Estado Libre Asociado de Puerto Rico). La autora entiende que "la espiritualidad se integra al repertorio del profesional de ayuda a través de un proceso tanto didáctico como vivencial", proveyendo de herramientas para aumentar la empatía y la receptividad tanto en el estudiante como en el profesional. Distingue un contenido espiritual, refiriéndose a todo tipo de material simbólico (contenido) con un significado idiosincrásico (proceso) para un usuario o un grupo. Amplía,

La espiritualidad es una realidad de vida que no puede ser negada. Ayuda a construir significado y propósito en la vida a un nivel más profundo. Requiere, de los profesionales y los estudiantes, respeto, tolerancia y aceptación hacia la diversidad y la sensibilidad cultural-espiritual (Fernández, 2015, p. 285).

En el caso de la Argentina los estudios son prácticamente nulos, vacancia que no sólo ocurre en el Trabajo Social local. En disciplinas como la psiquiatría y la psicología se ha reconocido recientemente que el concepto de espiritualidad ha sido sistemáticamente excluido del estudio de la salud mental, siendo -no obstanteuna dimensión clave que otorga sentido a la experiencia del vivir y fundamental a la hora de enfrentar la enfermedad y la muerte (Bianchi, 2013).

Pavón, Giménez y Rico (2014) proponen "Lo emocional y lo espiritual en el Trabajo Social. Una aproximación holística al campo profesional". Calvo y Reynoso (2003) contribuyen incorporando el enfoque gestáltico como herramienta en la práctica cotidiana, procurando de manera pionera, conjugar la actitud gestáltica y la tarea socioterapéutica del Trabajador Social. Para las colegas, el abordaje profesional es definido como socioterapia en el contexto de un proceso de aprendizaje psico-social que tiene en cuenta a la persona y la relación dinámica de la misma con su entorno. Tiempo antes, Armas y Kirchner de Mercado (1994) editan "Intuición y razón en Trabajo Social. Armonía de dos mundos' (1994) reuniendo los aportes del Método Silva de control mental y la psicorientología, junto a contribuciones de la psicología, la lógica y la ontología. Las autoras reconocen la extrañeza y reserva de los trabajadores sociales frente a estas nuevas aperturas, muchas veces tildados de locos o sectarios, las que son interpretadas como búsquedas personales que se preservan en los círculos de allegados. Elaboran una propuesta de intervención profesional basada en el "ser desde nuestro 
interior para una mejor calidad de vida, es hacer el camino del yo al nosotros" (Armas \& Kirchner de Mercado, 1994, p. 4). Recuperan aportes de algunos visionarios como Sela Sierra quien ya había iniciado esa búsqueda en los conversatorios Buscando el Tao del Trabajo Social, e invitan a "atreverse a mirar adentro y no tardarán en ver cómo se pueden integrar los últimos adelantos de la ciencia con la fe", propiciando un Trabajo Social "con grados crecientes de conciencia apropiada a un concepto holístico, en donde interactúa el cuerpo, la mente y el universo" (Armas y Kirchner de Mercado, 1994, p. 4).

De manera tangencial, también se integran perspectivas inscriptas en la tradición del Trabajo Social humanista que todavía está presente en diferentes programas de Trabajo Social en las universidades argentinas (Hermida, 2015). Estos enfoques se reflejan en el trabajo de Ander-Egg (2011) a través de una compilación reciente sobre el llamado neo-humanismo y la práctica crítica y liberadora, así como en Di Carlo (1997) quien integra los principios del existencialismo y el humanismo. Sin embargo, hay controversia; a pesar de ser autores fundantes de la disciplina en el país, las críticas a sus trabajos son severas, hasta el punto de considerar al humanismo como un obstáculo epistemológico para la investigación e intervención del Trabajo Social (Hermida, 2015). La polémica radica en el debate del humanismo/ antihumanismo de mediados del siglo $X X$, que parece actualizarse en el giro poscolonial.

Uno de los aspectos más discordantes se inscribe en la relación entre espiritualidad y cambio social, vistos históricamente como realidades antinómicas, disociando el cambio personal y el cambio social:

En el pasado, esta llevó a que muchas personas acogieran la perspectiva de la espiritualidad como una ideología o falsa conciencia -la religión como el opio del pueblo-, y que incluso fuera considerada como un obstáculo para el cambio y la transformación social [...] Ello condicionó el diálogo entre -por un lado- los megarelatos que alimentaron la ideología del progreso durante casi más de dos siglos, a partir del surgimiento de la ciencia moderna y del fenómeno de la llustración -y que desplazaron el monopolio del rol moralizador cumplido por las distintas religiones..., y por otro lado el surgimiento de nuevos tipos de espiritualidad con características laicas, agnósticas, híbridas o sincréticas, entre otras denominaciones posibles, y que integran en su visión de mundo dimensiones referidas a la vida en común y al bien de la comunidad (Equipo Editorial Polis, 2004, p. 1) 
El Trabajo Social argentino en particular, en su lucha por superar las raíces hispano-católicas de la asistencia social, sostuvo un carácter contradictorio, a diferencia de otros países donde la filantropía permitió la laicización de la asistencia sin excluir lo religioso. Se mantuvo de esta manera la tensión entre espiritualidad/ religiosidad y cambio social.

\section{Metodología de la investigación}

\section{Diseño}

Con el objeto de explorar la relación entre espiritualidad y Trabajo Social, desarrollamos un abordaje de carácter cualitativo con fines exploratorios $y$ descriptivos, con base en un diseño flexible y dinámico, por cuanto se desarrollan conceptos, intelecciones y comprensiones partiendo de los datos que se recogen y retroalimentan los presupuestos como los avances (Robson, 2002; Taylor \& Bodgan, 1996). La tarea de investigación incluyó un sondeo preliminar mediante entrevistas abiertas y diálogos informales mantenidos con trabajadores sociales; una revisión de literatura de las ciencias sociales y humanas, particularmente desde el año 2000 cuando -en base a Toniol (2015)- el tema presenta un creciente interés global con impacto en América Latina, para desarrollar luego -en una segunda fase- la recolección de información original mediante un cuestionario.
Estrategias de recopilación de información

En base a los aspectos emergentes de la búsqueda bibliográfica, basada en artículos y libros, informes de investigación y trabajos técnicos, se elaboró un cuestionario estructurado, anónimo y autoadministrado con el objeto de acercarnos a las visiones de los estudiantes. Cuenta con cuatro secciones en las que se desplegaron once preguntas: a) edad y sexo del respondente, y participación social por tipo de organización, como indicador de preocupación hacia los otros (rol de agencia); b) posicionamiento inicial ante la propuesta de una práctica profesional sensible a las creencias; c) la espiritualidad en la formación universitaria; y d) concepción del otro y finalidad de la intervención profesional. Se procuró acceder a información descriptiva acerca de aspectos de la formación, más también en línea con los supuestos que guiaron el acercamiento al tema, presumiendo que las concepciones respecto de la finalidad de la intervención como de la otredad podían explicar una mayor o menor apertura hacia la espiritualidad.

\section{Participantes}

Se aplicó durante los cursos de 2016 y 2017, a 137 estudiantes que estaban finalizando los estudios para obtener su grado de licenciatura en Trabajo Social en la Universidad Nacional de La Matanza (Buenos Aires, 
Argentina) ${ }^{1}$. La muestra intencional incluyó a los estudiantes que se encontraban en el aula y mostraron interés manifiesto en participar. La ausencia de indiferencia y rechazo a la propuesta connota inquietud en torno de la cuestión. A pesar de su naturaleza no probabilística, resulta adecuada para la recopilación de datos en muestras con características específicas, y que -por tal razónproporcionan información valiosa a los fines de la investigación.

La iniciativa reunió a estudiantes que pasaron por un proceso de socialización educativa similar. La proximidad de la graduación agregó estímulos y preocupaciones compartidas con respecto al trabajo profesional que se visualizan prontos a desempeñar. La muestra fue predominantemente de sexo femenino ( $n=125)$, de 30 años en promedio, con una mayoría en el rango de 20-25 años $(n=60)$. Provienen de la región Oeste de la Provincia de Buenos Aires (área de influencia

\footnotetext{
1 "La Universidad financiada íntegramente con recursos estatales, es de origen comparativamente reciente, y ofrece la formación en Trabajo Social como carrera fundacional desde 1989. Está localizada en la Municipio de La Matanza. Es el distrito con mayor población de la Provincia de Buenos Aires, con un total de 2.037.428 habitantes (INDEC: proyección 2015, basado en el censo del 2010), ubicado en un área de 325,71 km2 y distribuido en 484.909 hogares. También es el municipio con el mayor crecimiento de población intercensal (41,5\%, 2001-2010); el segundo porcentaje más alto de población que vive en viviendas precarias $(5,2 \%)$ y una tasa de analfabetismo significativa del 2,24\% (en comparación con la tasa media de analfabetismo del 1,4\% de la provincia)".
}

de la Universidad), la que por sus características demográficas refleja cabalmente el carácter multiétnico y pluricultural que presenta la Argentina, como resultado del mestizaje y las corrientes migratorias, pasadas y actuales. Sus localidades dibujan un paisaje con núcleos urbanos de alta concentración y centros comerciarles que coexisten con barrios precarios y enclaves marginales. Sus familias se ubican mayormente en sectores socioeconómicos medios y bajos, y cuyos ingresos monetarios provienen del empleo público, el ejercicio profesional y cuentapropistas en el sector servicios y construcción (Observatorio Social, Universidad Nacional de La Matanza, 2017).

Procedimiento para el análisis de la información

Los datos obtenidos fueron tabulados y procesados con la ayuda del Statistical Package for Social Sciences (SPSS, versión 24.0), a fin de describir y valorar las opiniones y creencias de los estudiantes. Finalizado el proceso reflexivo que propició el cuestionario, se consultó a los participantes acerca de su percepción ante la temática. El 65,7\% ( $n=90)$ se mostró interesado, un $16.8 \%(n=23)$ se sintió confundido, y un $7.3 \%(n=10)$ satisfecho con la propuesta. 
Consideraciones éticas

El cuestionario incluyó un preámbulo en el que se indican los objetivos y características de la investigación en la que se inscribe, los beneficios que reportará la información que se obtenga, el carácter voluntario, el tratamiento anónimo y la confidencialidad de los datos, indicando las personas responsables $y$ formas de contacto.

\section{Visiones de los estudiantes avanzados}

\section{Movilización espiritual}

El grado de movilización espiritual fue analizado en relación con las ideas acerca de los fines de la intervención y de sujeto. Encontramos que la espiritualidad no estaba tan distante, sino que formaba parte del discernimiento de los participantes. La mayoría entendía que los trabajadores sociales debían estar preparados para desarrollar una práctica sensible a las creencias espirituales, religiosas o filosóficas sostenidas tanto por los usuarios como por colegas, supervisores o líderes comunitarios y políticos: un $82.5 \%(n=113)$ acordaba fuertemente, mientras que un $16.8 \%$ $(n=23)$ lo hacía medianamente.

Al cotejar esta disposición favorable hacia la espiritualidad con los sentidos de la transformación social, notamos que la controversia teórica entre espiritualidad (habitualmente relacionada con un cambio interior centrado en el individuo) y justicia social (habitualmente relacionada con un cambio exterior centrado en los colectivos) se replica en la visión de los participantes. Están aquellos quienes creen que dicha oposición puede ser superada, y su articulación promovida como un doble objetivo de la intervención social, a diferencia de aquellos quienes dudan sobre ello y comprenden que ambas constituyen metas diferentes. Estas posiciones pueden atribuirse en parte a la concepción que se imparte en la educación profesional respecto de los fines del Trabajo Social y las dimensiones del cambio social, por lo general oscilando entre niveles micro y macrosocial, según se muestra en la Tabla 1. 
Tabla 1. Fines de la intervención profesional

Tipos de fines

Derechos de ciudadanía.

Satisfacción de necesidades

Bienestar psico-social $39 \quad 28.5$

Autonomía

No sabe

No contesta

Total

Adicionalmente, puede incidir en la tensión espiritualidad-justicia social, la concepción de sujeto que el profesional sostenga. Entre las opciones ofrecidas en el instrumento, la mayoría de las respuestas se concentraron en la idea de un ser con necesidades (Io que abre otro interrogante referido al alcance de la noción: necesidades biológicas, psicológicas, sociológicas, culturales, espirituales, etc.), como en un sujeto de derechos, poniendo de relieve la actual preponderancia del sujeto jurídico, genérico y abstracto (Ver Tabla 2).

\section{Tabla 2. Concepción de sujeto}

\section{Tipos de sujeto}

Ser con necesidades

Sujeto de derechos

Sujeto en riesgo

Sujeto esencialmente incompleto

Sujeto vulnerable

Sujeto interdependiente

Ninguna

No sabe

No contesta

Total
N

64

41

6

$14 \quad 10.2$

$5 \quad 3.6$

$1 \quad 0.7$

$4 \quad 2.9$

$1 \quad 0.7$

$\begin{array}{ll}1 & 10.7\end{array}$

$137 \quad 100.0$ 
La espiritualidad como dimensión del diagnóstico y la intervención

La mayoría de los participantes reconocieron que la espiritualidad es una dimensión que debe ser incluida en el diagnóstico social, aunque distinguiendo sus diversas manifestaciones. Las opciones se concentraron en atender la espiritualidad en tanto parte de las estrategias que las personas desarrollan para enfrentar problemas de salud, reducir el malestar y alcanzar la cura, como en los sentidos de la vida y el buen vivir (Ver Tabla 3).

\section{Tabla 3. Manifestaciones de la espiritualidad en el diagnóstico social}

\section{Tipos de manifestaciones}

En las formas religiosas

En las formas no-religiosas

En los sentidos de la vida y el buen vivir

En rituales, ceremonias e íconos

En las explicaciones del sufrimiento y la muerte

En las explicaciones de las causas últimas

En las estrategias que se desarrollan para

enfrentar el malestar y la enfermedad

No sabe

No contesta

Total

\section{N}

40

19

59

45

47

6

5

10

291
20.6

1.7

3.4

13.7

6.5

20.3

15.5

16.2

\section{1}

100.0
Respecto de la espiritualidad en la intervención, la relación también fue señalada positivamente. En el dominio de la práctica del trabajador social, la espiritualidad es un atributo que hace parte del respeto por la autonomía del usuario; también es vista como un factor de resiliencia, de cooperación con los sistemas sociales de apoyo; y como un elemento que moviliza a su defensa cuando es causa de opresión y discriminación por razones de fe, creencias o estilos de vida (Ver Tabla 4). 
Tabla 4. Manifestaciones de la espiritualidad en la intervención social

Tipos de manifestaciones

N

$\%$

Como factor de resiliencia

Como aspecto de la clínica

Ante actitudes discriminatorias por razones de fe o estilos de vida.....

En el uso de tradiciones folk y expresiones

espirituales, por su poder de comunión

Cuando la evidencia científica muestra su eficacia

En las estrategias de cooperación con los sistemas de apoyo social

Al enfrentar un dilema ético

En el respeto a la autonomía del otro 25.4

No lo reconoce.

No sabe

No contesta

Total

La espiritualidad en los contenidos curriculares y la bibliografía de apoyo

La presencia de la espiritualidad en los contenidos de los programas de estudio se consideró escasa en un 59.9\% ( $n=82$ ) de las respuestas, mientras un $19.7 \%(n=27)$ indica que es nula, y un $19 \%(n=26)$ opina que el tema está presente.

Respecto de la existencia de la literatura profesional disponible, las opiniones se distribuyeron de manera similar, con un 52.6\% ( $n=72)$ que encuentra escaso material de apoyo, un $24.1 \%$ ( $n=33$ ) no encuentra material, y solo un $12.4 \%(n=17)$ halla disponible.

Entre aquellos participantes que entienden que tales contenidos debieran incluirse en la formación (79.6\%; $n=109$ ), algunos de ellos (46\%; $n=63$ ) lo relacionan con habilidades para saber escuchar la historia personal, evaluar barreras y fortalezas, identificar recursos espirituales disponibles para el cambio, como para promover prácticas de autocuidado. En menor medida, los participantes hallan relación con valores de no-juzgamiento y respeto por la diversidad (17.5\%; $n=24)$. En un $16.8 \%$ ( $n=23$ ) los contenidos se identifican como parte de la base de conocimiento de la disciplina, en lo atinente a la relación entre el cambio social, las culturas y sistemas de creencias (Ver Tabla 5). 
Tabla 5. Contenidos educativos: habilidades, valores y base de conocimiento disciplinar

Tipos de contenidos

Como base de conocimiento

Como habilidades para saber escuchar

Como valores de no juzgamiento

No sabe

No contesta

No corresponde

Total
100.0

\section{Discusión}

Hallamos en la nueva generación de trabajadores sociales una disposición proclive a incorporar la espiritualidad en el ejercicio profesional. Cuanto menor es la edad del respondente mayor ha sido la apertura a los interrogantes planteados. El grado de participación en organizaciones sociales no ha influenciado la posición respecto a la espiritualidad y el Trabajo Social. En todo caso puede explicar una tendencia hacia distintas concepciones en torno de la finalidad profesional, como presupuesto para abordar la espiritualidad. Quienes sostienen que Trabajo Social debe estar preparado para desarrollar una práctica sensible a la dimensión sociocultural, se han mostrado mayoritariamente inclinados a ubicar la finalidad de la intervención en el plano de la ciudadanía política (41,5\%; $n=47)$ así como en el plano del bienestar psicosocial (28,3\%; n = 32), sentido que da cuenta de una transformación en términos personales como sociales.

Las opiniones han sido más favorables cuando se piensa en la espiritualidad en términos de habilidadesy valores de no juzgamiento; en menor medida cuando se trata de incorporarla al núcleo duro del conocimiento disciplinar. Al respecto, entendemos que sin su incorporación a la base conceptual, se mantienen las dificultades para superar: i) la ambivalencia histórica entre un extremo colectivista y otro individualista; ii) el carácter contradictorio y el rechazo a lo religioso/espiritual en la constitución de la identidad del Trabajo Social argentino; $y$ iii) el nivel de indeterminación e incompletud del conocimiento teórico utilizado por el Trabajo Social, lo que deriva en una permanente negociación de los significados entre los actores profesionales y no profesionales que participan en el campo de la acción social. 
Quienes consideran al usuario como un ser que tiene necesidades, fortalezas y experiencias, han mostrado una respuesta positiva respecto de la inclusión de la espiritualidad en los contenidos curriculares (53,2\%; $n=$ 58). En cambio, quienes lo han definido como sujeto de derechos, ciudadano genérico, el apoyo desciende (25,6\%; $n=28)$, aparece una posición negativa $(5,5 \% ; n=6)$ y de desconocimiento $(5,5 \% ; n=6)$.

Por su parte, la insuficiencia y vacancia que la temática registra tanto en el background teórico como instrumental caracteriza un camino de dificultades para el estudiante que quiere profundizar en esta dimensión. La necesidad de incorporar la espiritualidad y su relación con el bienestar resulta más clara y precisa cuando se trata del momento del diagnóstico social, y más difusa a la hora de incluirla en el plan de intervención.

En comparación con otros estudios, la escasa producción bibliográfica disciplinar en habla hispana y la ausencia de contenidos asociados, coincide con lo hallado en otras realidades. La falta de preparación dentro del currículo educativo de Trabajo Social, en uno de los mayores obstáculos (Moss, 2011; Gilligan \& Furness, 2006; Gotterer, 2001). De manera similar a otros resultados, la religión y la espiritualidad son importantes para la vida de los estudiantes y las personas en general, aunque se expresa de manera más imprecisa como tópicos de interés en la intervención profesional (Sheridan, 1999). En sintonía con el estudio de Muñoz-García y Ojeda (2018) también encontramos un interés de los estudiantes por la espiritualidad en los aspectos prácticos, y en menor medida en aspectos teóricos/reflexivos. La sensibilización sobre el tema es una conclusión compartida con otras investigaciones, con diferencias en los estadios de incorporación a la formación práctica. Para ello, debe cumplirse con el prerrequisito de contar con profesores capacitados (Bhagwan, 2013).

\section{Conclusiones}

La espiritualidad en el bienestar y la calidad de vida se han instalado en las ciencias sociales y humanas, lo que provee de nuevos flujos de conocimientos y matrices de análisis. Los estudiantes consultados se han mostrado receptivos y movilizados ante estos contenidos. La literatura coincide en la importancia que adquiere la temática para la formación en Trabajo Social: mejora la sensibilidad a la diversidad y al pluralismo cultural en una sociedad globalizada, así como las competencias teóricas y técnicas, no sólo en razón de las actuales posiciones de los organismos que 
marcan tendencia en materia de calidad de vida, sino en especial, atendiendo a la realidad de profesionales y usuarios que experimentan una pluralización de prácticas, saberes y agentes (Krmpotic \& Ponce de León, 2017). Asimismo, hemos notado como el tópico presenta un carácter desestabilizador en la medida que actualiza antiguas disyuntivas profesionales y revela los fundamentos ideológicos del Trabajo Social.

Este estudio representa una de las primeras investigaciones empíricas acerca de la espiritualidad en la formación en Trabajo Social en Argentina, desde la perspectiva estudiantil. Si bien sus resultados se limitan a estudiantes avanzados de una universidad nacional, el perfil del alumnado refleja el actual modo de vida urbano y el carácter multiétnico y pluricultural del país. La temática se manifiesta como una preocupación concreta, pero se integra con debilidades al universo del Trabajo Social. El interés no se ha exteriorizado aún en los contenidos curriculares ni en la agenda de los eventos profesionales, con una incipiente producción bibliográfica local. Dado que se trata de un área de vacancia requiere de mayor investigación.

La espiritualidad implica una actitud hacia la vida, una búsqueda hacia la transformación, una capacidad humana que permite trascender la vivencia personal, experimentar lo sagrado del universo y buscar el sentido de la vida, por lo que Trabajo Social no puede quedar ajeno al análisis de estos rasgos que hacen a la condición humana. Sostener la línea de investigación, y ampliar los canales de divulgación científica, académica y profesional constituyen metas de corto y largo plazo en orden a desarrollar un conocimiento especializado en torno del núcleo diversidad culturalespiritualidad-bienestar. De esta manera, el Trabajo Social podrá potenciar sus aportes respecto de: i) los problemas de comunicación causados por la falta de un lenguaje común, como por diferentes códigos de comportamiento en las interacciones con los servicios profesionales; ii) las diferencias culturales en las relaciones de parentesco, crianza, convivencia y satisfacción de necesidades; y iii) la interpretación de derechos y obligaciones de ciudadanía con el objeto de superar las barreras que dificultan la adaptación del sistema de servicios sociales a las necesidades de los sujetos.

\section{Limitaciones del estudio}

Además de los aspectos derivados de la naturaleza no probabilística de la muestra, como del carácter estructurado y autoadministrado del cuestionario, la investigación a futuro debiera incluir otras universidades a fin 
de ampliar el panorama de conjunto, y contrastar resultados con abordajes de las opiniones y creencias mediante instrumentos más flexibles tanto individuales como grupales.

\section{Agradecimientos}

Como integrante del proyecto PROINCE-PIDC A-203 (UNLaM), la Dra. Elsa Viviana Barrón colaboró en la elaboración del instrumento, como en la tabulación y procesamiento de los datos.

\section{Declaración de Conflicto de intereses}

Las personas autoras declararon que no hay conflictos de interés potenciales con respecto a la investigación, autoría o publicación en este artículo.

\section{Declaración sobre lenguaje inclusivo}

El uso específico de algún género gramatical en este artículo no supone un lenguaje sexista, invisibilizador ni excluyente.

\section{Financiamiento}

El estudio se llevó a cabo en el marco del proyecto "La dimensión cultural en la resolución de conflictos vitales en escenarios diversos" PROINCE-PIDC A-203 (2016-2017), financiado por la Universidad Nacional de La Matanza, Argentina.

\section{Referencias}

Ander-Egg, E. (2011). Humanismo y Trabajo Social. México: Facultad de Trabajo Social, Universidad Autónoma de Sinaloa,.

Armas, M. E., \& Kirchner de Mercado, A. (1994). Intuición y razón en Trabajo Social. Armonía de dos mundos. Buenos Aires: Humanitas.

Barrón, E. (2017). Trabajo Social, corporalidad y creencias religiosas en contextos confesionales. En J. Algranti, M. Mosqueira y D. Setton (Eds.), La institución como proceso: configuraciones de lo religioso en las sociedades contemporáneas. IX Conferencia sobre Ciencias Sociales y Religión (pp. 170-177). Buenos Aires: CEIL-CONICET.

Berger, P. (Ed.). (1999). The desecularization of the world. Resurgent religion and world politics. Michigan: Ethics and Public Policy Center and Wm. B. Eerdmans Publishing Co.

Bhagwan, R. (2013). Spirituality in social work in South Africa: Insights from a survey with academics. International Social Work, 56(3), 276-289.

Bianchi, R. (2013). Psiquiatría, psicología y espiritualidad. Buenos Aires: Tribunales Editorial. 
Cáceres, A., Hoyos, A., Navarro, R. \& Sierra G. (2008). Espiritualidad hoy: una mirada histórica, antropológica y bíblica. Theologica Xaveriana, 58(166), 381-408.

Calvo, L., \& Reynoso, L. (2003). TS y enfoque gestáltico. Una propuesta holística para la práctica cotidiana. Buenos Aires: Espacio Editorial

Canda, E. (2009). Spiritual diversity in Social Work practice: the heart of helping. New York: Oxford University Press.

Cervantes, C. (Ed.). (2011).

Espiritualidad y política. Barcelona: Kairós.

Crisp, B. (2008). Social Work and Spirituality in a Secular Society. Journal of Social Work, 8(4), 363-375.

De la Iglesia, P. (2011). Espiritualidad socialmente comprometida. En C. Cervantes (Ed.), Espiritualidad y política (pp. 175-187). Barcelona: Kairós.

\section{Di Carlo, E. (1997). El paradigma} humanista como sustancia del trabajo social: el trabajo social y el paradigma humanista dialéctico. Alternativas: Cuadernos de Trabajo Social, 5(1), 281-311.

Dominelli, L. (2004). Culturally competent social work: A way toward international anti-racist social work? En L. Gutierrez, M.
Zuniga \& D. Lum (Eds.), Education for Multicultural Social Work

Practice: Critical Viewpoints and

Future Directions (pp. 281-294) Alexandria, VA: Council on Social Work Education.

Equipo Editorial (2004). Espiritualidad y cambio social: ¿realidades antinómicas? Polis[online] 8, 1-14. Recuperado de http://polis.revues. org/5954

Fernández, R. (2015). La espiritualidad en la academia: creando espacios. En L. Morales Alejandro, G. Negrón Velázquez, R. Estremera Jiménez y M. Marqués Llompart (Eds.), Espiritualidad y Trabajo Social: controversias y oportunidades (pp. 282-301). Departamento de Trabajo Social de la Universidad de Puerto Rico: San Juan de Puerto Rico.

Foucault, M. (2008). La arqueología del saber. Buenos Aires: Siglo XXI.

Furman, L., Benson, P.W., Canda,

E., \& Grimwood, C. (2005). A Comparative International Analysis of Religion and Spirituality in Social Work: A Survey of UK and US Social Workers. Social Work Education, 24(8), 813-839.

Gilligan, P., \& Furness, S. (2006). The Role of Religion and Spirituality in Social Work Practice: Views and Experiences of Social Workers 
and Students. British Journal of

Social Work, 36(4), 617-637.

Gotterer, R. (2001). The Spiritual

Dimension in Clinical Social Work Practice: A Client Perspective.

Families in Society, 82(2), 187193.

Hermida, M. (2015). El Humanismo como obstáculo epistemológico del trabajo social: Una lectura crítica del texto Humanismo y Trabajo Social de Ezequiel AnderEgg. Cátedra Paralela, 12(1), 85-119.

Hick, S. (Ed.). (2008). Mindfulness and Social Work: Paying Attention to Ourselves, our Clients and Society. Chicago: Lyceum.

Jacobs, C. (1997). On spirituality and social work practice. Smith College Studies in Social Work, 67(2), 171-175.

Janigová, E., \& Bednarova, V. (2015). La importancia de los indicadores de espiritualidad de la calidad de vida a comienzos del siglo XXI. En L. Morales Alejandro, G. Negrón Velázquez, R. Estremera Jiménez y M. Marqués Llompart (Eds.), Espiritualidad y Trabajo Social: controversias y oportunidades (pp. 203-251). Departamento de Trabajo Social de la Universidad de Puerto Rico: San Juan de Puerto Rico.
Kearney, M. (1990). Spiritual Pain. The Way, 30(1), 47-54.

Krmpotic, C. (2018). Movilización espiritual en perfiles profesionales. Reflexiones a partir de un caso ilustrativo. (En)clave Comahue, 24(1), 143-160.

Krmpotic, C. (2016). La espiritualidad como dimensión de la calidad de vida. Exploraciones conceptuales de una investigación en curso. Scripta Ethnologica, XXXVIII, 105-122.

Krmpotic, C., \& Ponce de León, A. (2017). Diez enunciados para pensar la identidad profesional en Trabajo Social. Una invitación al debate internacional en los albores del siglo XXI. Cuadernos de Trabajo Social, 30(1), 135-147.

Krmpotic, C., \& Ponce de León, A. (2015). Training specialists in Forensic Social Work. An experience of cross-cultural education in Latin America. Social Dialogue. International Association of Schools of Social Work, 3(12), 6-9.

Lindsay, R. (2002). Recognizing Spirituality: The Interface between Faith and Social Work. Crawley: University of Western Australia Press. 
Moñivas, A., García, G., \& García De Silva, R. (2012). Mindfulness: práctica, constructo, teoría, método e intervención ¿nuevo paradigma? En E. Sobremonte de Mendicuti (Ed.), Epistemología, teoría y modelos de intervención en Trabajo Social. Reflexión sobre la construcción disciplinar en España (pp. 301-310) Bilbao: Universidad de Deusto.

Morillo, M., Galan, J., \& Llanos, F. (2017) Actitudes de las Enfermeras ante los Cuidados Espirituales y Religiosos en un Hospital General. Index de Enfermería, 26(3),152-156.

Moss, B. (2005). Religion and Spirituality. Lyme Regis: Russell House Publishing.

Muñoz-García, A., \& Ojeda, Á. (2018). ¿Están los estudiantes españoles de trabajo social interesados en recibir formación en competencias espirituales? Educació Social. Revista d'Intervenció Socioeducativa, 69(1), 115-130.

Myers, D. (2008). Religion and human flourishing. En M. Eid \& R. Larsen (Eds.), The science of subjective well-being. New York: Guilford.

Observatorio Social (2017).

Matanza en cifras: Principales indicadores socioeconómicos. Universidad Nacional de La Matanza. Recuperado de http://observatoriosocial. unlam.edu.ar/descargas/6_ EmpleoenLaMatanza.pdf.

Ortiz, M. (2007). Dilemas Éticos de la Espiritualidad en Trabajo Social. Quinta Conferencia de Trabajo Social Forense, Universidad interamericana, Recinto Metropolitano, Puerto Rico (inédito).

Pavón, P., Gimenez, A., \& Rico, M. (2014). Lo emocional y lo espiritual en el Trabajo Social. Una aproximación holística al campo profesional. Margen: Revista de Trabajo social y Ciencias Sociales, 74, 1-9.

Perea, M. (2016). Espiritualidad, logoterapia y trabajo social: Aportaciones para una práctica holística del trabajo social. Documentos de Trabajo Social, 58(1), 146-161.

Perrin, D. (2007). Studying Christian Spirituality. New York: Routledge.

Sheridan, M., \& Amato-von Hemert, K. (1999). The role of religion and spirituality in Social Work education and practice: a survey of students views and experiencies. Journal of Social Work Education, 35(1), 125-141.

Sperry, L., \& Shafranske, E. P. (Eds.). (2005). Spiritually Oriented Psychotherapy. Washington, 
D.C.: American Psychological Association Publishing.

Toniol, R. (2015). Espiritualidade que faz bem. Pesquisas, políticas públicas e práticas clínicas pela promoção da espiritualidade como saúde. Sociedad y Religión, 43(1), 110-143.

Torrent, R. (2011). Poner conciencia a la política. En C. Cervantes (Ed.), Espiritualidad y política (pp. 139160) Barcelona: Kairós.

World Health Organization (2012). WHOQOL Spirituality, Religiousness and Personal Beliefs (SRPB) Field-Test Instrument. Geneva: WHO. 